\title{
The effect of training on plasma L-carnitine metabolism in purebred Arabian horses
}

\author{
W. Kędzierski ${ }^{1}$ \\ University of Life Sciences, Faculty of Veterinary Medicine, \\ Department of Animal Biochemistry and Physiology \\ Akademicka 12, 20-033 Lublin, Poland
}

(Received 22 May 2009; revised version 25 January 2010; accepted 19 August 2010)

\begin{abstract}
The aim of this study was to evaluate how training affects plasma total, free and esterified L-carnitine concentrations in horses. The study was done on a total of 32 healthy, trained purebred Arabian horses. The 32 horses comprised two groups. In the first group, 12 stallions and 6 mares three-year-old involved in race-training were studied at rest. In the second group, 14 stallions were studied during a typical race or endurance training session. Jugular venous blood samples were collected to determine lactic acid (LA), free fatty acids, glycerol, triacylglycerides (TG), and the total esterified and free L-carnitine contents. The race training session caused a significant increase in blood LA and plasma esterified L-carnitine, glycerol, and TG levels. It also caused a decrease in the plasma free L-carnitine concentration determined just after the end of exercise.

Compared with endurance training, high-intensity racing exercise increased the plasma esterified L-carnitine concentration and decreased that of free L-carnitine according to training intensity.
\end{abstract}

KEY WORDS: horses, carnitine, endurance, exercise test, lipid, race

\section{INTRODUCTION}

Racing or athletic ability is the main selection criterion in the breeding of warmblood horses. Exercise performance requires a high potential for lipid oxidation. This process is dependent on the rate of fatty acid transport into the mitochondria. It also requires the presence of L-carnitine and its undisturbed metabolism.

\footnotetext{
${ }^{1}$ Corresponding author: e-mail: witold.kedzierski@up.lublin.pl
} 
L-carnitine (3-hydroxy-4N-trimethylaminobutyrate) is a water-soluble quaternary amine. It is present in muscle cells as a free form of L-carnitine and as fatty acid and acetyl esters. The primary function of L-carnitine is to transport long-chain fatty acids into the mitochondria through the mitochondrial membrane. Synthesis of acetylcarnitine plays an important role in the regulation of the mitochondrial acetyl-CoA/CoA ratio. It equalizes the utilization of carbohydrates and fatty acids in energy production. In horses that are exercising, the intramuscular concentration of free L-carnitine decreases and the acetylcarnitine content increases equivalently, whereas the total L-carnitine pool remains unchanged (Foster and Harris, 1987a; Carlin et al., 1990). After short intensive exercise periods, the levels of intramuscular free L-carnitine and its esters return to their resting values in about 30 min (Harris and Foster, 1990).

The L-carnitine content in the blood plasma of trained horses has not been studied much. It is known that the total plasma L-carnitine content determined at rest increases during the course of the day. It reaches a peak level during the afternoon (Zeyner and Harmeycr, 1999). The study of Foster and Harris (1987b) indicated that the concentration of plasma free L-carnitine measured immediately after intensive exercise remained unchanged, but dropped significantly during recovery. A similar tendency was observed in male athletes after a maximal intensity workout during a treadmill ergometry test (Nuesch et al., 1999). However, medium-intensity exercise involved an increase in plasma acetylcarnitine determined after $90 \mathrm{~min}$ (Bordin et al., 1992). Also, the changes in the plasma free L-carnitine concentration while exercising were different compared with the changes recorded in muscles. On the other hand, Schulpis et al. (2009) noticed a correlation of acylcarnitine with adrenaline blood levels in athletes. Westermann et al. (2008) stated that in Standardbred horses, the plasma acylcarnitine profile reflected the skeletal muscle carnitine metabolism following exercise. The reason for changes in the plasma acylcarnitine/free carnitine ratio during exercise remains under discussion. The plasma concentration of various carnitine forms following exercise in purebred Arabian horses has not been assessed.

The aim of this study was to evaluate the effects of high-intensity exercise required for race training and for endurance training, on the plasma L-carnitine profile in purebred Arabian horses. This was to be evaluated on a background of parameters of lipid metabolism.

\section{MATERIAL AND METHODS}

A group of 32 healthy, trained purebred Arabian horses were studied. The study was accepted by the Local Ethics Review Committee for Animal Experimentation 
of the Agricultural University in Lublin and was conducted according to the European Community regulations concerning the protection of experimental animals. During the experiment, the horses were trained and kept at the racetrack (Warsaw Shiżewiec, Poland) or at a local training centre. The horses were fed with typical feeds used for race horses in Poland. The diet composed of oats and supplemented with about $200 \mathrm{ml}$ of soya oil and a mineral-vitamin premix without L-carnitine. To establish the plasma total, free, and esterified L-carnitine levels, as well as indices of lipid metabolism according to the training process, two experiments were conducted.

In experiment 1 , a group of 18 three-year-old purebred Arabian horses (12 stallions and 6 mares) trained for the racetrack was studied while at rest during the consecutive phases of their training process. Blood samples were collected three times: the first in December, which was before the start of their training process, then in June and in November. Blood samples were always collected in the afternoon. The horses were on a conventional race training schedule, as described below. The regular training programme started in January. The first month of training consisted of walking and trotting while saddled. The next month, the horses cantered for a distance of about $500 \mathrm{~m}$ at a speed of $6 \mathrm{~m} / \mathrm{s}$. The distance and speed were increased as the horse showed improvement. After about four months of training, all of the studied horses competed in official races. By that time, they had been training five days a week. The daily training routine consisted of a warm up, a gallop with a mean speed of $12-16 \mathrm{~m} / \mathrm{s}$ to a distance of $800-1200 \mathrm{~m}$ and cooling down in a horse walker. The intensity of the training was consistent with the principles of a trainer preparing the horses for races.

In experiment 2, purebred Arabian stallions were studied during a typical training session. The horses were divided into two groups. The first group of 8 stallions, 3-5 years old, was being trained to race. Prior to the start of this study, this group of horses was in the training programme described above. The second group consisted of 6 stallions, 5-7 years old, that were being prepared for endurance riding. Prior to the start of this study, these horses had been in conventional endurance training. They had been trotted $1-2 \mathrm{~h}$ and cantered 5-10 min. This had been done three times a week. On the day of the study, the race training session consisted of a warm-up trot, galloping $1800 \mathrm{~m}$ at a mean speed of $10 \mathrm{~m} / \mathrm{s}$, and a $5-\mathrm{min}$ trot. The endurance horses trotted $30 \mathrm{~min}$ and galloped 5-10 $\mathrm{min}$ with a mean speed of 6-8 $\mathrm{m} / \mathrm{s}$. Three jugular venous blood samples were taken from each horse into test tubes containing EDTA. These blood samples were obtained while at rest, just after the end of a training session, and $30 \mathrm{~min}$ after the end of exercise. After sampling, $0.01 \mathrm{ml}$ of blood was put into a cuvette of lactic acid (LA) test kits. The remaining blood was immediately centrifuged for plasma separation. 


\section{Blood analysis}

Blood LA levels were determined using Dr. Lange's (Berlin, Germany) enzymatic test (Wandrup et al., 1989). The assay was done within $4 \mathrm{~h}$ from sampling and the results expressed as $\mathrm{mmol} / \mathrm{l}$. Plasma free fatty acids (FFA), glycerol and triacylglyceride (TG) levels were determined by enzymatic methods using Boehringer Mannheim (Darmstadt, Germany), Roche (Mannheim, Germany) and Cormay (Lublin, Poland) tests, respectively, and expressed as $\mu \mathrm{mol} / \mathrm{l}$. The plasma free L-carnitine content was assayed by the Roche (Mannheim, Germany) enzymatic UV test after plasma deproteinization in $0.6 \mathrm{~mol} / \mathrm{dm}^{3}$ perchloric acid and following neutralization by the addition of $1.2 \mathrm{~mol} / \mathrm{dm}^{3}$ potassium carbonate solution. The plasma total L-carnintine concentration was measured using the same Roche test, but first plasma was prepared as described by Benamou and Harris (1993) with a minor modification. To induce hydrolysis of esterified L-carnitine, $0.5 \mathrm{ml}$ of plasma was added to $0.25 \mathrm{ml} 1.5 \mathrm{~mol} / \mathrm{dm}^{3} \mathrm{KOH}$ and incubated for $20 \mathrm{~min}$ at $37^{\circ} \mathrm{C}$. Then $0.75 \mathrm{ml}$ of $0.6 \mathrm{~mol} / \mathrm{dm}^{3}$ perchloric acid was added and after $10 \mathrm{~min}$ incubation in an ice-bath the samples were centrifuged at $3000 \mathrm{~g}$. At this point, $0.1 \mathrm{ml}$ of $1.2 \mathrm{~mol} / \mathrm{dm}^{3}$ potassium carbonate was dropped into $1 \mathrm{ml}$ of supernatant to neutralize the low $\mathrm{pH}$ of the obtained mixture. The solution obtained this way was used as the fuel substrate for measurement of total L-carnitine. The level of esterified L-carnitine was calculated as the difference between the plasma total and the free L-carnitine concentrations expressed as $\mu \mathrm{mol} / 1$.

\section{Statistical analysis}

All values were given as means and standard deviations (SD). For all the analysed blood and plasma parameters, comparisons between groups (race training vs endurance training) were performed using the unpaired Student t-test (Microsoft Excel XP). The effect of the effort and training processes were assessed done using the Tukey test (Statistica 6.0, Software).

\section{RESULTS}

Experiment 1. In the group of 18 purebred Arabian horses, the resting plasma TG level was significantly lower in the last sampling as compared with those observed before the start of the training process (Table 1). Data obtained after four months of race-training were also lower than those obtained at the very beginning of this study. This decrease did not reach statistical significance because of the high individual variability and consequential high standard deviation. The plasma 
glycerol and FFA as well as total, free and esterified L-carnitine concentrations did not differ significantly following the training process (Table 1).

Table 1. Plasma total, free and esterified carnitine, free fatty acids (FFA), glycerol and triacylglycerides (TG) levels in 18 three years old horses studied at rest during consecutive phases of race-training, means $\pm \mathrm{SD}$

\begin{tabular}{lccc}
\hline $\begin{array}{l}\text { Plasma indices, } \\
\mu \mathrm{mol} / \mathrm{l}\end{array}$ & $\begin{array}{c}\text { Samples taken in } \\
\text { December, before the } \\
\text { start of training process }\end{array}$ & $\begin{array}{c}\text { Samples taken } \\
\text { in June, } \\
\text { after four months } \\
\text { of race-training }\end{array}$ & $\begin{array}{c}\text { Samples taken } \\
\text { in November, } \\
\text { at the end } \\
\text { of race-scason }\end{array}$ \\
\hline Total carnitine & $36.5 \pm 7.11$ & $38.9 \pm 6.06$ & $37.8 \pm 8.60$ \\
Free carnitine & $28.1 \pm 9.18$ & $31.4 \pm 2.03$ & $26.7 \pm 5.70$ \\
Esterified carnitine & $8.4 \pm 7.20$ & $7.5 \pm 6.84$ & $11.1 \pm 8.34$ \\
FFA & $81.6 \pm 60.1$ & $110 \pm 80.2$ & $92.0 \pm 63.8$ \\
Glycerol & $24.0 \pm 22.6$ & $18.6 \pm 20.7$ & $35.7 \pm 58.6$ \\
TG & $350 \pm 90.3^{\text {a }}$ & $292 \pm 124^{\text {ab }}$ & $303 \pm 62.1^{\mathrm{b}}$ \\
\hline a.b - means in rows with different superscripts differ at $\mathrm{P} \leq 0.05$ &
\end{tabular}

Experiment 2. Data obtained during the training session are shown in Table 2. The endurance training session induced statistically significant increases in blood LA and plasma glycerol levels determined just after the end of exercise as compared with resting values. The race-training session caused a significant increase in blood LA and plasma esterified L-carnitine, glycerol and TG levels.

Table 2. Values of determined indices in horses during two types of training sessions

\begin{tabular}{|c|c|c|c|c|c|c|}
\hline \multirow{2}{*}{$\begin{array}{l}\text { Type of } \\
\text { exercise } \\
\text { Plasma indices }\end{array}$} & \multicolumn{3}{|c|}{ Endurance training session, $n=6$} & \multicolumn{3}{|c|}{ Race training session, $n=8$} \\
\hline & at rest & $\begin{array}{l}\text { just after } \\
\text { the exercise }\end{array}$ & $\begin{array}{l}\text { after } 30 \\
\text { min rest }\end{array}$ & at rest & $\begin{array}{c}\text { just after } \\
\text { the exercise }\end{array}$ & $\begin{array}{l}\text { after } 30 \\
\text { min rest }\end{array}$ \\
\hline \\
\hline total camitine & $36.5 \pm 1.72$ & $36.3 \pm 5.73$ & $33.9 \pm 4.45$ & $37.0 \pm 3.72$ & $35.1 \pm 2.13$ & $34.3 \pm 3.61$ \\
\hline free carnitine & $28.3 \pm 4.62$ & $25.0 \pm 4.86$ & $23.1 \pm 3.79$ & $28.0 \pm 4.8^{\mathrm{a}}$ & $15.8 \pm 2.5^{\mathrm{b} *}$ & $13.2 \pm 3.5^{b *}$ \\
\hline $\begin{array}{l}\text { esterified } \\
\text { carnitine }\end{array}$ & $8.2 \pm 1.22$ & $11.3 \pm 1.32$ & $10.8 \pm 2.58$ & $9.0 \pm 1.66^{\mathrm{a}}$ & $19.3 \pm 1.4 I^{\mathrm{b} *}$ & $21.1 \pm 3.57^{b *}$ \\
\hline glyccrol & $14.3 \pm 10.6^{\mathrm{a}}$ & $84.9 \pm 44.3^{b}$ & $21.6 \pm 20.4^{a}$ & $24.4 \pm 17.1^{\mathrm{a}}$ & $93.3 \pm 74.0^{b}$ & $153 \pm 270^{\llcorner *}$ \\
\hline TG & $362 \pm 131^{a}$ & $391 \pm 153^{\mathrm{a}}$ & $314 \pm 72.5^{b}$ & $272 \pm 90.3^{a}$ & $344 \pm 110^{b}$ & $263 \pm 112^{\mathrm{a}}$ \\
\hline $\mathrm{LA}, \mathrm{mmol} / \mathrm{l}$ & $1.27 \pm 0.48^{a}$ & $2.46 \pm 1.41^{\mathrm{b}}$ & $1.42 \pm 0.39^{a}$ & $0.89 \pm 0.28^{\mathrm{a}}$ & $5.61 \pm 4.45^{\mathrm{b} *}$ & $2.07 \pm 3.61^{\circ}$ \\
\hline
\end{tabular}

The race training session also caused a decrease in the plasma free L-carnitine concentration determined just after the end of exercise as compared with the data obtained at rest. A 30 min rest was insufficient for LA, free and esterified L-carnitine contents to return to resting levels. Plasma glycerol measured $30 \mathrm{~min}$ after the 
end of exercise was significantly higher than just after it. Comparison between the two groups of horses indicated that the values of blood LA determined just after exercise were significantly higher in race horses than in endurance horses. Plasma esterified L-carnitine levels measured just after exercise and then again $30 \mathrm{~min}$ later, and also glycerol determined after $30 \mathrm{~min}$ rest were significantly higher in horses involved in race training than in endurance horses. Only plasma free L-carnitine values were lower in race horses than in endurance horses when measured just after the exercise period as well as after a $30 \mathrm{~min}$ rest.

\section{DISCUSSION}

The problem of the effect of various levels of exercise intensity on encrgy metabolism in Purebred Arabian horses is especially noteworthy. Horses of this breed are used in two different ways. Young Arabian horses take part in races on the race track whereas adult horses are used in endurance rides. These two types of exercise require completely different predispositions. Also, participation in a race or endurance competition should be preceded by the appropriate type of training.

The data obtained in experiment 1 while the horses were at rest was collected during one season of their race-training. The concentrations of plasma glycerol and FFA as well as total, free and esterified L-carnitine were essentially unchanged with training. Only the plasma TG level dropped significantly. This at-rest data indicates that the training process did not influence the plasma carnitine level but did influence lipid metabolism regulation. In work-load or stress conditions, under the influence of increased sympathetic nervous activity, TG are mobilized from adipose tissue and FFA and glycerol are liberated into the bloodstream. Under resting conditions, FFA are carried bound to plasma albumins, but during the increased rate of lipolysis, the plasma FFA concentration can increase 10-fold and the buffering ability of the albumins to bind can be impaired. About twothirds of the liberated plasma FFA is absorbed by hepatocytes and esterified again. Afterwards, the synthesized TG are packed in very low density lipoproteins and released into the bloodstream again. In light of this fact, the lower plasma TG level shown in this work after about one-year-long training, reflects the predominance of FFA utilization over the TG synthesis in the horses studied.

It is possible to state that conventional race-training decreases the TG level in blood plasma of race horses. A similar effect was described in a previous study of purebred Arabian horses (Kędzierski and Podolak, 2002). In the experiment described by Munoz et al. (2002) even a two-month training period for Arabian horses had a significant effect on the regulation of energy metabolism in these animals. These observations can be explained by the progressive development of 
the mechanism for controlling lipid metabolism with training.

The horses studied in this part of the experiment did not show any changes in plasma L-carnitine levels. The capacity for biosynthesis of L-carnitine in young animals is limited and cannot meet the requirement of horses whose feed contains only a small amount of carnitine. Thus, the possibility and effectiveness of carnitine supplementation were tested. Supplementation slightly increased the level of this parameter in plasma (Zeyner and Harmeyer, 1999). It also had a limited effect on horse metabolism as well as performance (Rivero et al., 2002; Van Weyenberg et al., 2009).

In the study of young Thoroughbred horses, the plasma endocrine free L-carnitine content significantly increased with the onset of adolescence and amounted to $8 \mu \mathrm{mol} / \mathrm{l}$ in yearlings, $15 \mu \mathrm{mol} / 1$ in two-year-olds, and more than 26 $\mu \mathrm{mol} / \mathrm{l}$ in horses aged 3-6 years (Foster et al., 1989). In two-year-old Thoroughbred horses, a two-fold increase in the plasma free L-carnitine level was found after one year of training (Foster et al., 1989). Moreover, the value of this parameter was higher in trained than in untrained three- to six-year-old horses (Foster et al., 1989). On the other hand, six-month-long training did not affect plasma free and esterified L-carnitine concentrations in two-year-old Standardbred horses (Westermann et al., 2008). Also, the increase in the plasma carnitine level under the influence of training was observed in Thoroughbred horses by Foster et al. (1989) but not in Standardbred horses by Westermann et al. (2008). Moreover, at the very beginning of this study, the Arabian horses investigated had a high plasma L-carnitine level which was found to be characteristic of adult horses.

In experiment 2 the blood LA level increased about 2-fold in the group which underwent endurance training. It increased 6-fold in horses subjected to race-training, crossing in this group the anaerobic threshold which amounts to $4 \mathrm{mmol} / 1$ (McMiken, 1983). The determination of LA is used in practice to evaluate the relative intensity of exercise in horses (Davie and Evans, 2000). The more intense the exercise, the more LA production increases. The changes in other investigated parameters except total L-carnitine depended on the intensity of exercise. They were more expressed during a more intensive race-training session. The increase in plasma glycerol and TG levels and also in the contribution of the esterified form in total L-carnitine content were higher in race training sessions than in endurance training sessions. A positive correlation between horse blood LA and changes in the plasma glycerol concentration measured after exercise was described previously (Kędzierski et al., 2007). In galloping horses, an increase in plasma glycerol level was spectacularly high when it was measured $30 \mathrm{~min}$ after the end of exercise (Kędzierski, 2007). This observation confirms the suggestion that in race horses during the recovery period after intensive galloping, lipolysis and glycerol secretion into blood continue. The increase in plasma TG 
concentration while exercising is also characteristic for horse metabolism and was observed previously in Arabian horses (Podolak et al., 2004). The source of plasma TG during exercise is the process of estrification of FFA extracted from the bloodstream by the liver cells.

The plasma total L-carnitine content was not influenced by the applied exercise. Similar results were observed in other horse and human studies (Bordin et al., 1992; Iben et al., 1999). The race-training session did, however, differentiate the plasma L-carnitine fraction levels. The exercise increased the esterified L-carnitine content with a simultancous decline in the free form. The results of Soop et al. (1988) indicated that the most likely site for plasma L-carnitine esterification is not muscle but the liver. While exercising, muscles mainly release the free form of L-carnitine and adipose tissue provides FFA. Enzyme carnitine acyltransferase I, which is present in hepatocytes, catalyses the binding of L-carnitine and fatty acids (the first step to $\beta$-oxidation). As a result of its activity, part of acylated L-carnitine is released into the bloodstream. This process persists as long as the lipolysis rate is enhanced. In the studied horses, the high level of plasma glycerol determined $30 \mathrm{~min}$ after the end of exercise indicates that lipolysis was still stimulated at that time. The elevated rate of lipolysis increased the amount of FFA involved in esterification with free L-carnitine. As a result, the production of esterified L-carnitine in the liver was also increased.

Under conditions of intensive exercise, there was an increase of not only the hepatic production of esterified L-carnitine, but also its secretion to the bloodstream. Probably, the ability of hepatocytes to utilize fatty acids in the mitochondria is limited in comparison with the FFA supply. An additional reason for limited FFA utilization in the liver can be the increase of the LA concentration. Within hepatocytes, LA is oxidized to pyruvate, which is converted to acetyl$\mathrm{CoA}$. An increased acetyl-CoA/CoA ratio has an inhibitory effect on the enzymes of $\beta$-oxidation and decreases FFA utilization. The process of binding L-carnitine and fatty acids, however, still continues. As a result, within liver cells, the amount of esterified L-carnitine increases and a part of that is liberated to the bloodstream. Also, the increase of an esterified form of L-carnitine in blood plasma can be an indicator of the decreased ability of the liver to utilize fatty acids during intensive exercise.

\section{CONCLUSIONS}

The present study provides evidence that one year-long race training did not influence the plasma level of total, free or esterified forms of L-carnitine in young purebred Arabian horses. Compared with endurance training, racing high- 
intensity exercise increased plasma esterified L-carnitine, and decreased the free

L-carnitine concentrations according to the intensity of the applied exercise.

\section{REFERENCES}

Benamou A.E., Harris R.C., 1993. Effect of carnitine supplement to the dam on plasma carnitine concentration in the sucking foal. Equine Vet. J. 25, 49-52

Bordin D., Bottecchia D., Bettini V., Aragno R., Sartorelli L., 1992. Effect of middle-intensity exercise on carnitine and beta-hydroxybutyrate plasmatic concentration in men and women. J. Sports Med. Phys. Fitness 32, 394-399

Carlin J.I., Harris R.C., Cederblad G., Constantin-Teodosiu D., Snow D.H., Hultman E., 1990. Association between muscle acetyl-CoA and acetylcarnitine levels in the exercising horse. J. Appl. Physiol. 69, 42-45

Davie A.J., Evans D.L., 2000. Blood lactate responses to submaximal field exercise test in Thoroughbred horses. Vet. J. 159, 252-258

Foster C.V.L., Harris R.C., 1987a. Formation of acetylcarnitine in muscle of horse during high intensity exercise. Eur. J. Appl. Physiol. Occup. Phys. 56, 639-642

Foster C.V.L., Harris R.C., 1987b. Changes in the free and bound carnitine in muscle with maximal sprint exercise in the Thoroughbred horse. In: J.R. Gillespie, N.E. Robinson (Editors). Equine Exercise Physiology 2. ICEEP Publications, Davis, CA, pp. 332-340

Foster C.V.L., Harris R.C., Pouret E.J.M., 1989. Survey of plasma free carnitine levels in 74 Thoroughbred horses at stud and training. Equine Vet. J. 21, 139-141

Harris R.C., Foster C.V.L., 1990. Changes in muscle free carnitine and acetylcarnitine with increasing work intensity in the Thoroughbred horse. Eur. J. Appl. Phys. Occup. Phys. 60, 81-85

Iben C., Moschitz E., Fehleisen B., 1999. Effect of L-carnitine supplementation on heart rate and some blood parameters in the eventing horse (in German). Wien. Tierärztl. Monatsschr. 86, 330-338

Kędzierski W., 2007. The correlations of blood lactic acid and glycerol levels in Purcbred Arabian horses during exercise tests. Ann. UMCS, Sect. DD 62, 46-52

Kędzierski W., Kowalik S., Janczarek 1., 2007. Influence of interval and endurance training on the level of chosen blood parameters and heart rates in trotters. Med. wet. 63, 1258-1261

Kędzierski W., Podolak M., 2002. Training Arabian horses and its effect on the level of biochemical indices related to the metabolism of carbohydrate and lipids. Med. wet. $58,788-791$

McMiken D.F., 1983. An energetic basis of equine performance. Equine Vet. J. 15, 123-133

Munoz A., Riber C., Santisteban R., Lucas R.G., Castejon F.M., 2002. Effect of training duration and exercise on blood-borne substrates, plasma lactate and enzyme concentrations in Andalusian, Anglo-Arabian and Arabian breeds. Equine Vet. J., Suppl. 34, 245-251

Nuesch R., Rossetto M., Martina B., 1999. Plasma and urine carnitine concentrations in well-trained athletes at rest and after exercise. Influence of L-carnitine intake. Drug. Exp. Clin. Res. 25, $167-171$

Podolak M., Kędzierski W., Janczarek I., 2004. Intense training of Arabian horses and its effect on the level of selected biochemical indices in their blood and heart rate. Med, wet. 60, 403-406

Rivero J.L., Sporleder H.P., Quiroz-Rothe E., Vervuert I., Coenen M., Harmeyer J., 2002. Oral L-carnitine combined with training promotes changes in skeletal muscle. Eqine Vet. J., Suppl. $34,269-274$ 
Schulpis K.H., Parthimos T., Papakonstantinou E.D., Tsakiris T., Parthimos N., Mentis A.F., Tsakiris S., 2009. Evidence for the participation of the stimulated sympathetic nervous system in the regulation of carnitine blood levels of soccer players during a game. Metabolism 58, 1080-1086

Soop M., Björkman O., Cederbland G., Hagenfeldt L., Wahren J., 1988. Influence of carnitine supplementation on muscle substrate and carnitine metabolism during exercise. J. Appl. Physiol. 64, 2394-2399

Van Weyenberg S., Buyse J., Janssens G.P.J., 2009. Increased plasma leptin through L-carnitine supplementation is associated with an enhanced glucose tolerance in healthy ponies. J. Anim. Physiol. Anim. Nutr. 93, 203-208

Wandrup J., Tvede K., Grinsted J., Jordening H., 1989. "Stat" measurements of L-lactate in whole blood and cerebrospinal fluid assessed. Clin. Chem. 35, 1740-1743

Westermann C.M., Dorland B., de Sain-van der Velden M., Wijnberg I.D., van Breda E., de GraafRoelfsema E., Keizer H.A., van der Kolk J.H., 2008. Plasma acylcarnitine and fatty acids profiles during exercise and training in Standardbreds. Amer. J. Vet. Res. 69, 1469-1475

Zeyner A., Harmeyer J., 1999. Metabolic functions of L-carnitine and its effects as feed additive in horses. A review. Arch. Tierernähr. 52, 115-138 